University of Wollongong

Research Online

Faculty of Engineering and Information

Faculty of Engineering and Information

Sciences - Papers: Part A

Sciences

$1-1-2013$

A novel approach to investigate surface roughness evolution in asymmetric rolling based on three dimensional real surface

H Xie

Tokyo Metropolitan University

K Manabe

Tokyo Metropolitan University

Z Y. Jiang

University of Wollongong, jiang@uow.edu.au

Follow this and additional works at: https://ro.uow.edu.au/eispapers

Part of the Engineering Commons, and the Science and Technology Studies Commons

Research Online is the open access institutional repository for the University of Wollongong. For further information contact the UOW Library: research-pubs@uow.edu.au 


\title{
A novel approach to investigate surface roughness evolution in asymmetric rolling based on three dimensional real surface
}

\begin{abstract}
Surface roughness affects the friction behaviour, stress distribution and surface quality; moreover this influence is more significant when scaling down the strip size. A novel approach has been proposed to investigate the surface roughness in asymmetric rolling based on Finite Element Analysis (FEA). Evolution of asperity flattening, contact area ratio and surface roughness is investigated under various simulation rolling conditions. The model reviewed numerical solutions for real surfaces with asperities of arbitrary shape and varying size and height distributions. The rolling characterization has also been identified for asymmetric rolling. The difference of change in surface roughness for upper and lower strip surface is large during rolling. The effect of the speed asymmetry factor on strip bending has been established. An increase of the speed asymmetry factor causes an increase in the strip curvature, while the force parameters decrease with increasing asymmetry factor. The speed asymmetric factor influences the strip bending significantly. It is the first attempt to carry out this kind of research in surface roughness based on real surface during asymmetric rolling of thin strip. 2013 Elsevier B.V.
\end{abstract}

\section{Keywords}

rolling, three, dimensional, real, asymmetric, roughness, investigate, surface, approach, novel, evolution

\section{Disciplines}

Engineering | Science and Technology Studies

\section{Publication Details}

Xie, H., Manabe, K. \& Jiang, Z. Y. (2013). A novel approach to investigate surface roughness evolution in asymmetric rolling based on three dimensional real surface. Finite Elements in Analysis and Design, 74 (October), 1-8. 


\title{
A novel approach to investigate surface roughness evolution in asymmetric rolling based on three dimensional real surface
}

\author{
H.B. Xie ${ }^{a, b}$, K. Manabe ${ }^{a}$ and Z.Y. Jiang \\ ${ }^{1}$ Department of Mechanical Engineering, Tokyo Metropolitan University, Tokyo 192-0397, Japan \\ ${ }^{2}$ School of Mechanical, Materials and Mechatronic Engineering, University of Wollongong, \\ Wollongong NSW 2522, Australia
}

\begin{abstract}
Surface roughness affects the friction behaviour, stress distribution and surface quality; moreover this influence is more significant when scaling down the strip size. A novel approach has been proposed to investigate the surface roughness in asymmetric rolling based on Finite Element Analysis (FEA). Evolution of asperity flattening, contact area ratio and surface roughness are investigated under various simulation rolling conditions. The model reviewed numerical solutions for real surfaces with asperities of arbitrary shape and varying size and height distributions. The rolling characterization has also been identified for asymmetric rolling. The difference of change in surface roughness for upper and lower strip surface is large during rolling. The effect of the speed asymmetry factor on strip bending has been established. An increase of the speed asymmetry factor causes an increase in the strip curvature, while the force parameters decrease with increasing asymmetry factor. The speed asymmetric factor influences the strip bending significantly. It is the first attempt to carry out this kind of research in surface roughness based on real surface during asymmetric rolling of thin strip.
\end{abstract}

Keywords: Surface roughness, real contact area, ultra-thin strip, asymmetric rolling, strip curvature

\section{Introduction}

Asymmetric rolling (ASR), a new promising process for the manufacturing of metal sheets and strips with small size, can be achieved by using rolls with unequal diameters, by prescribing different speeds of rotation to the rolls, by imposing different friction coefficients between the two rolls and the rolled strip, or by a combination of the above various factors [1]. Tribology plays a significant role for process feasibility and quality, especially in micro forming where the influence of the tribological condition is very essential due to an extensive increase in friction while scaling processes from macro to micro. Asperity flattening in surface roughness affects the friction behaviour at the interface and the transfer of surface roughness during rolling process [2 - 4]. Therefore the surface roughness research is extremely important in metal forming, especially in micro rolling.

A large amount of research work concerning surface roughness has been carried out in metal forming. Asperity flattening is a key point in research of surface roughness and friction. Y. Kinura et al [5] proposed surface asperity deformation under bulk plastic straining conditions. The velocity fields based on energy minimization method were applied to predict the actual metal flows. The surface roughing affects the asperity flattening [6, 7]. Contact mechanics of rough surfaces in multiple asperities [8] was investigated to study the effect of roughness distribution on the real contact area. The surface topography [9] was assumed as different model and the simulation results from the three-wavelength model are closer to experimental results. Combined effects of friction and macroscopic deformation on asperity flattening were also reported in Ref [10]. Recent work by Wilson and Sheu has investigated the effect of bulk plasticity on asperity flattening when the lay of the roughness is parallel to the bulk straining direction [11]. H.R. Le et al [4] presented analysis of 
surface roughness using the Fourier transform method in cold rolling. FEM simulation provided new tools for metal forming [12 - 16]. K. Manabe et al [12] reported validation of FE simulation based on surface roughness model in micro-deep drawing. Finite element simulations are performed based on the new friction model to enable more flexible modelling of friction behaviours [13]. For asymmetric rolling, Yeong-Maw Hwang et al. [1, 17] carried out analytical and experimental study on asymmetrical sheet rolling. As reduction and frictional factor increase, the relative bonding length increases. The asymmetric rolling parameters were also analysed in Refs [18, 19], and the strip curvature has been evaluated [20].

These researches have shown that the real areas of contact increase with the contact stress and strip plastic strain, and the dimension of strip body could influence asperity deformation. All these provide useful information for metal forming operations. However, for a number of reasons, most of these theories assumed asperities to be regularly distributed on the strip surface, and this is different from the real surface features of the rolled strip. In the miniaturization of thin strip rolling, the proportion of surface roughness of the materials to total thickness becomes larger with a decrease strip thickness, which should be considered as non-uniformity of thickness. In addition, surface roughness is thought to be the dominant process factor related to formability. The valley of surface roughness is also a source of stress concentration [21], resulting in defect formation or propagation

Based on the real strip surface, a novel finite element model of surface roughness has been developed in this study and the characterization of asymmetric thin strip rolling has also been identified. The comparison examines the evolution of real contact area, surface roughness and contact pressure distribution at the interface between rolls and the rolled thin strip under various rolling conditions. It is the first attempt to investigate the surface roughness evolution in asymmetric rolling based on real surface features.

\section{Asymmetric rolling analysis}

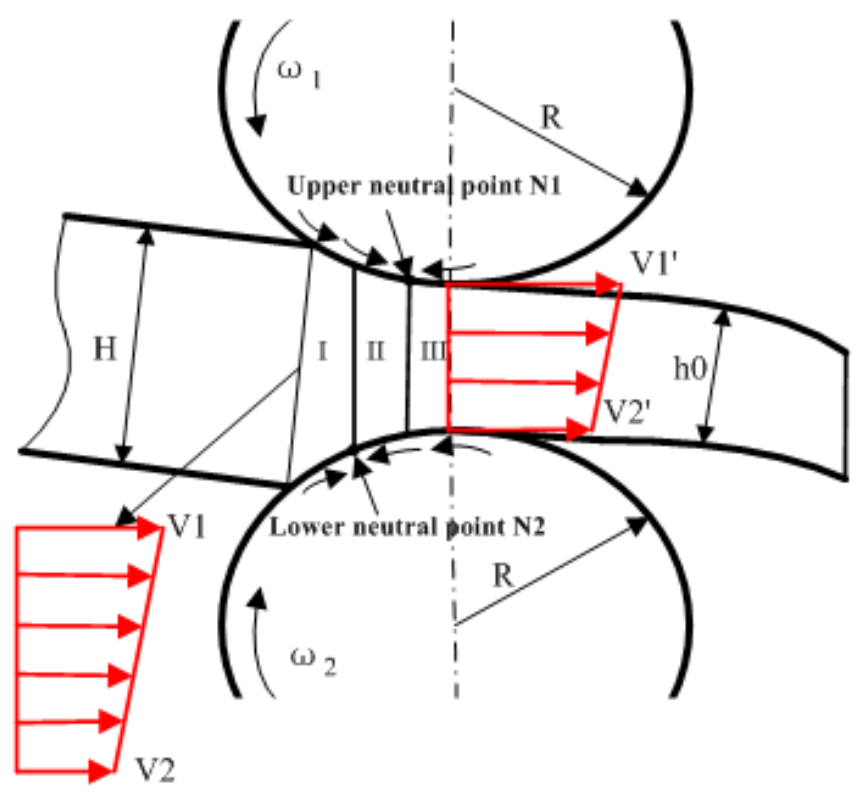

Fig. 1 Schematic illustrations of asymmetric rolling

Fig. 1 shows the schematic illustrations of asymmetric rolling where the speed of the upper roller is higher than that of the lower roller. The non-uniform distribution of longitudinal speed is 
significant across the strip thickness. Because of higher reduction of the upper strip layer resulted from higher speed, the upper neutral point is shifted along the rolling direction to near the deformation exit. Correspondingly the critical angle and forward slip are greater than that in symmetric rolling. An increase of the roll speed ratio will result in an increase in the length of the shear zone. At the same time, due to unchanged energy balance for asymmetric rolling in the vertical plane, the lower neutral point will be moved back to near the rolling entrance. Variation in these parameters increases the longitudinal speed of the strip near the upper roller and reduces the longitudinal speed of the strip near the lower roller in the exit. In this case, the critical angle, forward slip, and longitudinal speed of the strip near at the lower roller in the exit plane are reduced. This process improves productivity of the rolling operation in reducing of the rolling force, pressure, torque and improving properties of the strip surface. The neutral points are at different positions resulting in a cross shear zone between them.

As shown in Fig.1, the whole plastic deformation region at the roll gap is divided into three distinct regions according to the directions of the friction force exerted on the strip from the upper and lower rolls: zone I for the entrance region, zone II for the cross shear region (CSR) and III for the exit region. From the volume constancy of the material, the positions of the upper and lower neutral points $N_{1}$ and $N_{2}$, have the following relationship as [19]

$$
x_{N 1}=\sqrt{\forall_{A} x_{N 2}^{2}+\left(\forall_{A}-1\right) \frac{h_{0}}{R}}
$$

where, $\forall_{A}=\frac{\omega_{2}}{\omega_{1}}$ is speed ratio (speed asymmetric factor) of two rollers; $\mathrm{R}$ roller radius, mm; $h_{0}$ thickness after rolling, mm; $x_{N 1}$ and $x_{N 2}$ are the distances of upper and lower neutral point to the vertical symmetry plane of two rollers, respectively.

\section{Surface roughness modelling in FEM}

\subsection{Surface roughness modelling}

In order to simulate the rolling process accurately, appropriate models for material behaviour across a large range of size scales are necessary. Fig. 2 shows the three dimensional measured heights of surface roughness of the steel strip and the measured values were obtained from measurement system: 3D Laser Scanning Microscope, VK-X100. The rolling direction and rolling trace have also been identified in Fig 2. Note that the surface roughness profile seems in random and the characterization of higher height distribution is significant along rolling direction and shown in rolling trace, and the maximum relative height is around $5.8 \mu \mathrm{m}$. Fig. 3 shows the two dimensional surface profile in a certain length of $100 \mu \mathrm{m}$.

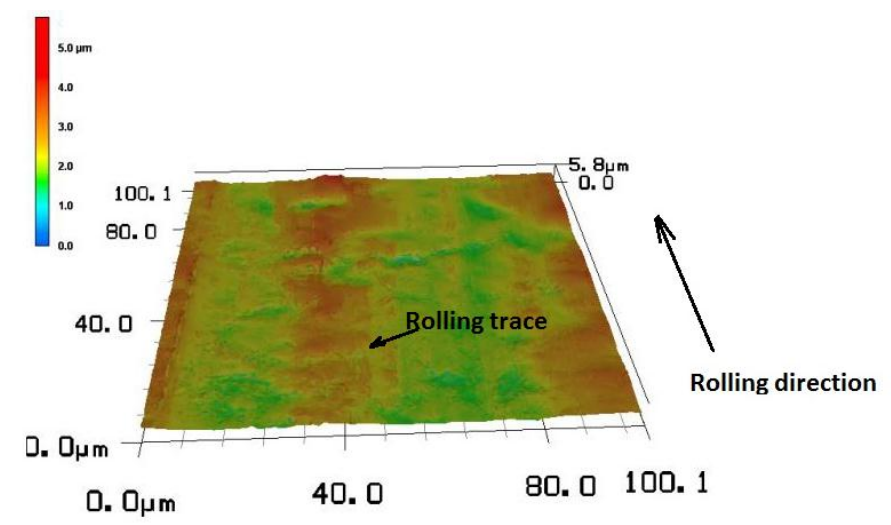

Fig. 2 Three dimensional measured heights of surface roughness. 


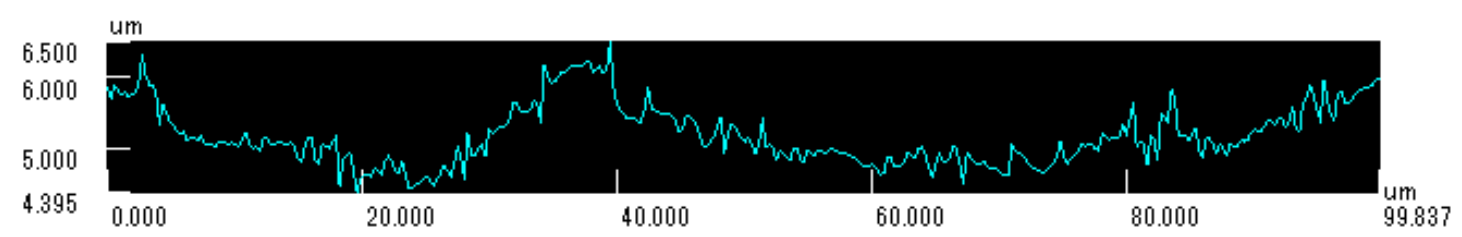

Fig. 3 Two dimensional surface profile

A continuous function is required to eliminate the need of integrating discrete functions during the numerical procedure. Fourier series makes it possible to describe non-smooth asymmetric distribution functions from which the accuracy of the evaluation depends on the number of expansions used $[9,22,10]$. However this kind of function still can not represent the real surface feature [23]. In this study, the surface roughness model based on the real measured value from Fig. 2 has been set up through the ANSYS Parametric Design Language (APDL) as shown in Fig. 4 (a). Fig. 4 (b) is the 3D meshing of one corner of the model where the three dimensional characterization of asperities is illustrated. The element type is 3D solid element. Mesh size is changed with the element position and the strip surface is meshed in much finer size.

(a)

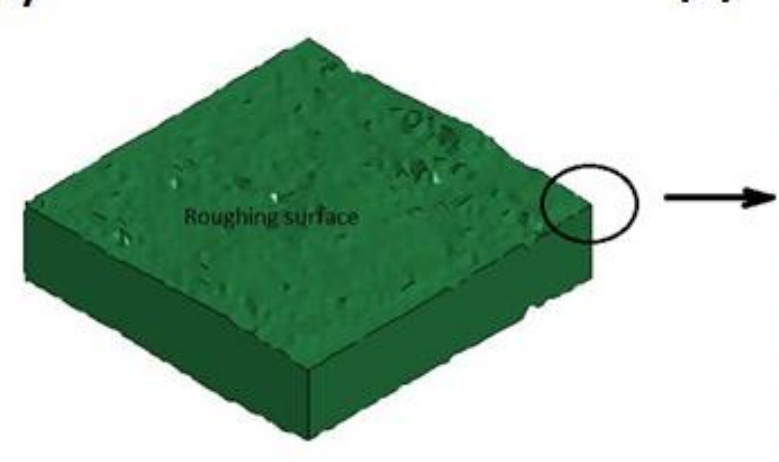

(b)

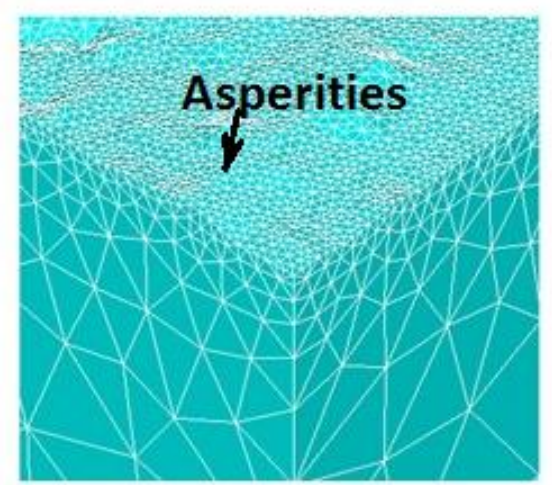

Fig. 4 Modelling surface roughness based on real surface roughness.

(a) Surface roughness modelling (b) 3D meshing of one corner of the model

\subsection{Simulation conditions}

The simulation was carried out with an explicit dynamic finite element code, LS-DYNA. The diameter of the two rolls was $125 \mathrm{~mm}$ and the rolled strip was selected for the analysis by utilising the three dimensions $200 \times 40 \sim 180 \times 1.5 \sim 2.0 \mathrm{~mm}$ in the rolling, transverse and thickness directions, respectively. Element types used for the roll and the rolled strip is SOLID164 (three dimensional 8 NODE solid element), and the finite element mesh in the rolled strip includes 165,380 nodes and 121,378 elements with refined elements around the surface roughness for improving the simulation accuracy and further surface roughness calculation after simulation. In the rolling process, the roll rotates with a fixed angular velocity, and the strip enters the roll with an initial velocity, and exits under the friction force. A surface-to-surface contact condition is defined between the roll and the strip. Solution termination time is $1.5 \mathrm{~s}$ and time steps for LS-DYNA include number of elements to plot of 100, translucency value of 0.9 and time step scale factor is 0.9 .

The rolled strip was assumed to be isotropic elastic-plastic, while the model of the work rollers was assumed to be linear-elastic material. Table 1 shows the mechanical properties of the work roller and rolled strip used in the simulation. The static and kinetic friction coefficients between the strip and roller were assumed to be 0.035 and 0.025 , respectively. The analysis of asymmetric 
rolling in the thin strip has been carried out within the present study with the aim of establishing the effect of the speed asymmetry factor, $\forall_{A}$, on rolling force, strip bending and surface roughness during the rolling process. In this simulation, the change of surface roughness of the roll can be ignored due to its higher hardness than that of the rolled strip as shown in Table 1. The roller surface was assumed to be smooth and the texture effect of the roll would not be taken into consideration.

Table 1 Mechanical properties of the work roller and rolled strip

\begin{tabular}{|c|c|c|}
\hline & Roll & Steel strip \\
\hline Material model & Elastic & Elastic-plastic \\
\hline Density, Kg/m ${ }^{3}$ & $7.85 \times 10^{3}$ & $7.85 \times 10^{3}$ \\
\hline Young`s Module MPa & $2.1 \times 10^{5}$ & $1.17 \times 10^{5}$ \\
\hline Poisson`s ratio & 0.3 & 0.3 \\
\hline Hardness & $800 \mathrm{HV}$ & $168 \mathrm{HV}$ \\
\hline Yield stress, MPa & & 250 \\
\hline
\end{tabular}

\section{Simulation result and discussions}

Analytical studies show that the real contact area between the rolled strip and the roller occurs at isolated points where the asperities came together. With increasing pressure, the plastic deformation fields around individual asperities merged. Due to surface roughness it occurs at discrete points, and the force between the bodies is the sum of the individual forces on contacting asperities which constitute the pressure distribution. Fig. 5 shows the schematic deformation model of asperities. It can be seen that the asperities flattening occurs near the contact interface between the roller and the rolled strip. When surfaces are loaded together under dry conditions or with boundary lubrication, most of the load is carried by the asperities in close contact. In these cases the friction between the roller and strip will be controlled by the growth of the true areas of contact.

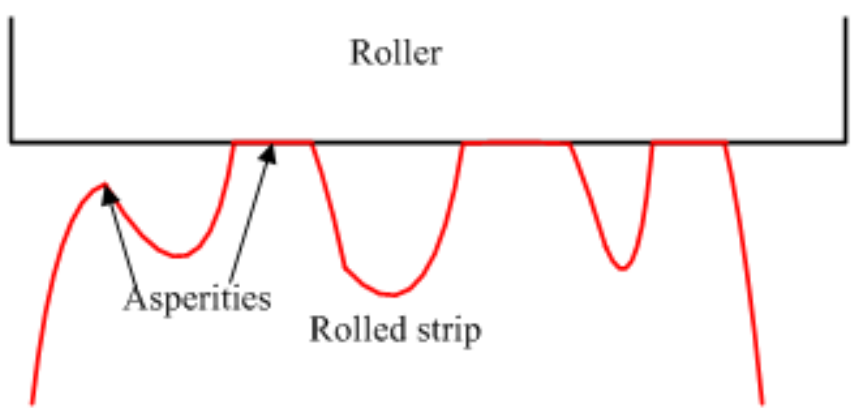

Fig. 5 Schematic deformation model of asperities.

Two factors complicate the picture of contact growth in rolling processes. Firstly, at the high pressures involved, there is interaction between contacting asperities and secondly the deformation of the rolled materials will affect the asperity deformation. Without any bulk deformation, the growth of contact area will be limited as the pressure increases. The effect of bulk plasticity was highlighted which showed how asperities were flattened when deforming short cylinders with bulk deformation directly below the asperities [24]. The area of contact ratio was increased in model asperities with bulk deformation of the substrate, and the rate of asperity flattening with bulk 
straining was related to the spacing of the asperities and to the difference in pressure between loaded and unloaded parts.

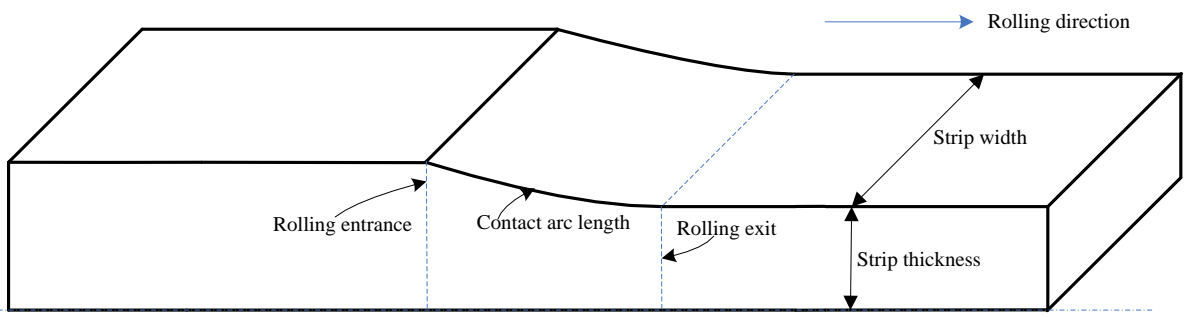

Fig. 6 A sketch of deformed half-thickness strip during cold rolling.

Fig. 6 shows a sketch of deformed half-thickness strip during cold rolling. The contact arc length existing rolling entrance and rolling exit is the contact length between the roll and the strip.

\subsection{Real contact area evolution}

The real area of contact can be defined as a function of the surface topography, material properties and interfacial loading conditions as shown in Eq.2.

$$
A_{r}=f(\text { Surface topography, material properties, loading conditions) }
$$

When two surfaces move relative to each other, the adhesion of these asperities and other sources of surface interactions contribute to friction force. A real contact region consists of contact spots, the total area of which is a small fraction of the nominal contact area which is the minimal connected region enclosing all the contact spots. Fig. 7 shows the real contact area under asymmetric rolling. The total contacting length of the profile, as well as the equivalent stress at each node contacting the roller is recorded. The former is used to calculate the contact area ratio, while the data of the equivalent stress are collected for further analysis. The randomness comes from the random nature of surface roughness, whereas the different sizes of spots occur due to the multiple scales of roughness.

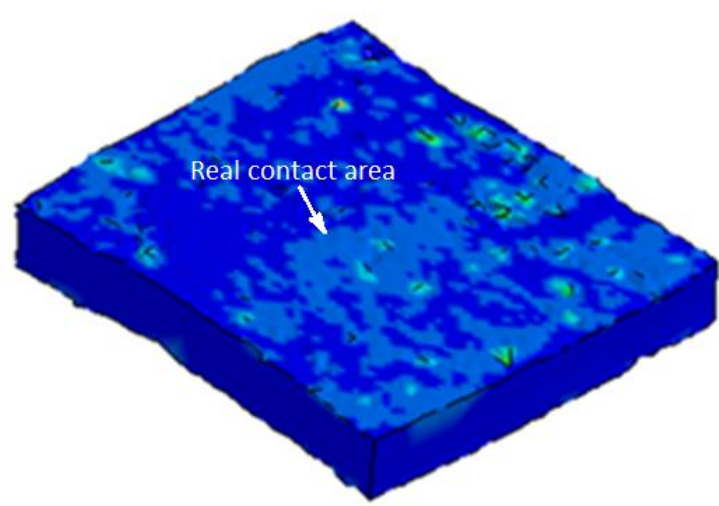

Fig. 7 Real contact area under rolling pressure.

For a given load, the size of spots depends on the surface roughness and the mechanical properties of the contacting materials. The existing spots will increase in size with an increase of rolling pressure, new spots will appear, and two or more spots may coalesce to form a larger spot. The calculation of real contact area ratio is listed below, 


$$
\alpha=\frac{A_{r}}{A_{a}}
$$

where, $A_{r}$ and $A_{a}$ are real contact area and apparent area, respectively.

Simulated surface roughness is considered to be a high frequency, wavelength of a calculated surface, which is characterized through average roughness $R_{a}$.

$$
R_{a}=\frac{1}{l} \int_{0}^{l}|Z(x)| d x
$$

where, $l$ is calculated length, and $Z(x)$ is the measured height from the simulation.

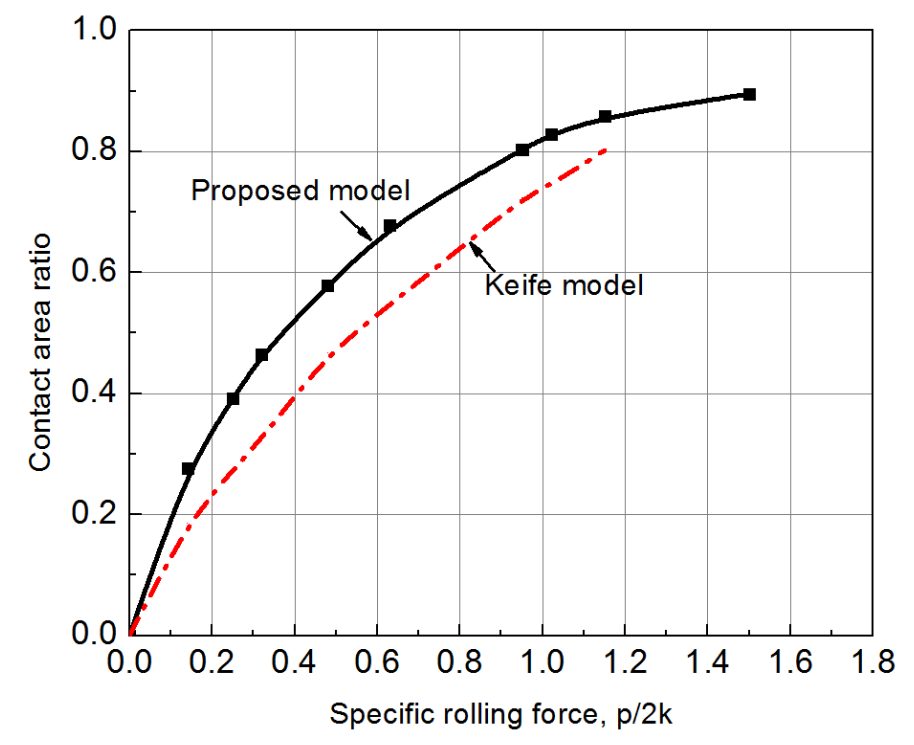

Fig. 8 Contact area ratio versus the specific rolling force.

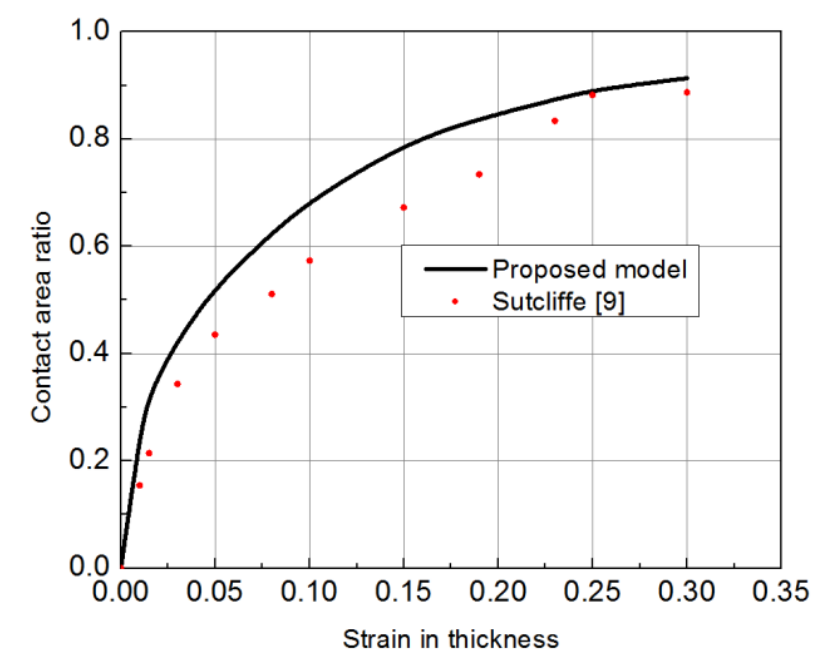

Fig. 9 Contact area ratio dependence on strain in thickness.

Fig. 8 compares the evolution of area of contact ratio with specific rolling force obtained by the FEM models and Keife's model [25]. Keife's model presented the creation of surface asperities considering the deformation hardening of work material and the elastic deformation of the roll, and the model can be used to study the influence of friction related process parameters such as lubricant 
viscosity and rolling speed. To obtain a general understanding, specific rolling forces applied are all normalized by over the yield stress in plain strain. It is clear that the contact ratio increases as a function of specific rolling force for the two models. The curve of present model is relatively close to that of Keife model with a maximum difference of $10.5 \%$ at $\mathrm{p} / 2 \mathrm{k}$ of 0.7 . The difference between present model and the Keife model apparently becomes larger before the specific rolling force reaches 1.2. Both curves have similar trends and the differences between two models might be explained as different asperity slopes and different methods being used in the simulations.

Real contact area fraction as a function of strip strain in simulation shown in Fig. 9 and Sutcliffe [9] experimental results are also plotted. The asperities on the surfaces to interact are in a nonuniform way, exerting forces on each other and leading to frictional forces. With an increase of strain in thickness, the contact area ratio increase accordingly. However, this trend is much more flattening when the contact area ratio reach near 0.9. In other words, the change of contact area ratio is insensitive with further increased strain in thickness.

\subsection{Surface roughness evaluation in FE simulation}

Fig. 10 shows the change of the surface roughness ratio with specific rolling force, and $R_{a}{ }^{\prime}$ is the roughness after flattening while $R_{a}$ is the initial roughness before rolling. Note that the roughness ratio decreases with an increase of specific rolling force because the roller flattens the asperities and peaks much more under higher rolling force.

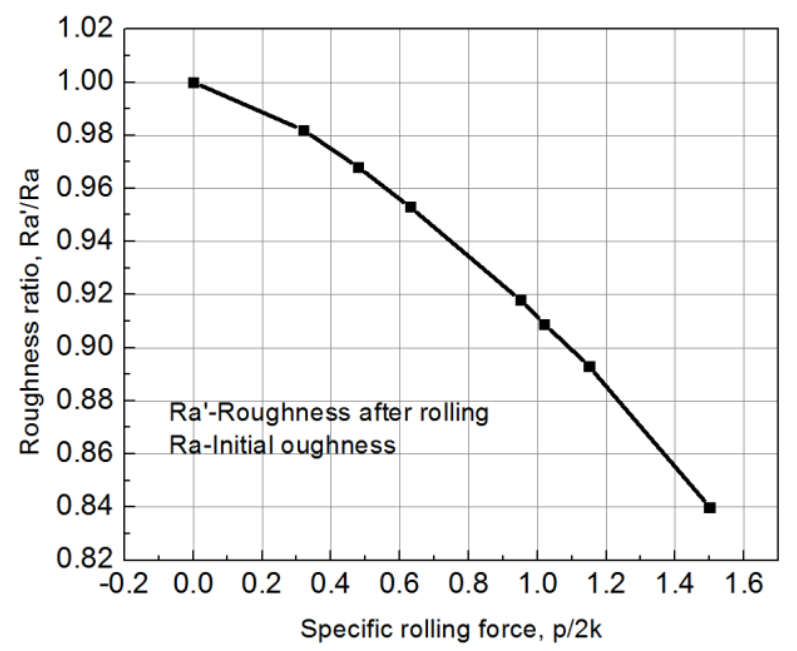

Fig. 10 Surface roughness change with specific rolling force.

Compared Fig. 9 with Fig. 10, it can be seen that when the strain in thickness reaches over 0.3 the contact area ratio tends to 1, a nearly completely flattened surface. However, the height of asperities still reduces with an increase of rolling pressure and this results in reduced surface roughness ratio in Fig. 10, while the contact area between the roll and rolled strip remains constant or less change depending on asperity shape as shown in Fig. 9. In other words, the contact area ratio is not in a strict monotone function with the change of surface roughness.

The surface roughness ratio of the upper side and the bottom side with strip thickness and speed ratio is shown in Fig. 11. Results reveal that the roughness ratio increases with strip thickness and decreases with speed asymmetric factor. Nevertheless, both sides of the strip surface have nearly conformed to the roll surface after the rolling. Since the surface roughness on both the incoming and outgoing strip is close to that of the rolls, any effect of surface roughness is overshadowed by scatter in the measurements. Nevertheless, there appears to be a slight increase in the surface roughness at very small, which may be associated with a breakdown of lubrication. The surface 
roughness difference between lower side and upper side of the rolled strip is higher in higher speed ratio than in lower speed ratio. In addition, this difference reduces with an increase of the rolled strip thickness.

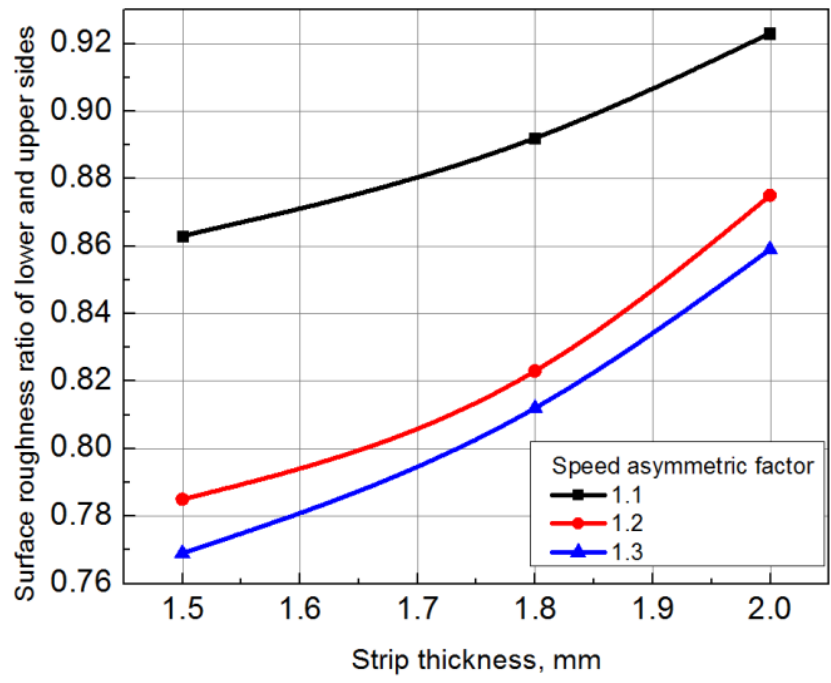

Fig. 11 Roughness amplitude of the rolled strip.

Fig. 12 shows the surface roughness versus pass reduction under different initial thicknesses. It can be seen that the surface roughness decreases with an increase of pass reduction and the decreasing of initial strip thickness. With the increasing rolling pass reduction, more plastic deformation will occur. The reprinting between the roller and the strip becomes more significant which results in the decreasing of the surface roughness of rolled strip.

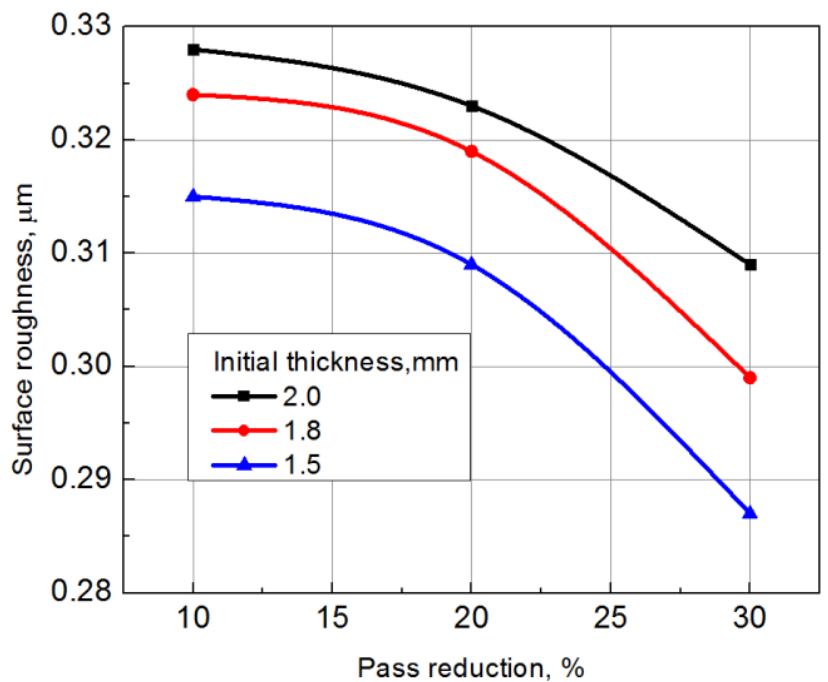

Fig.12 Surface roughness versus pass reduction under different initial thickness.

\subsection{Rolling characterization of asymmetric rolling}

The effect of rolling speed asymmetric factor is investigated in this section. 


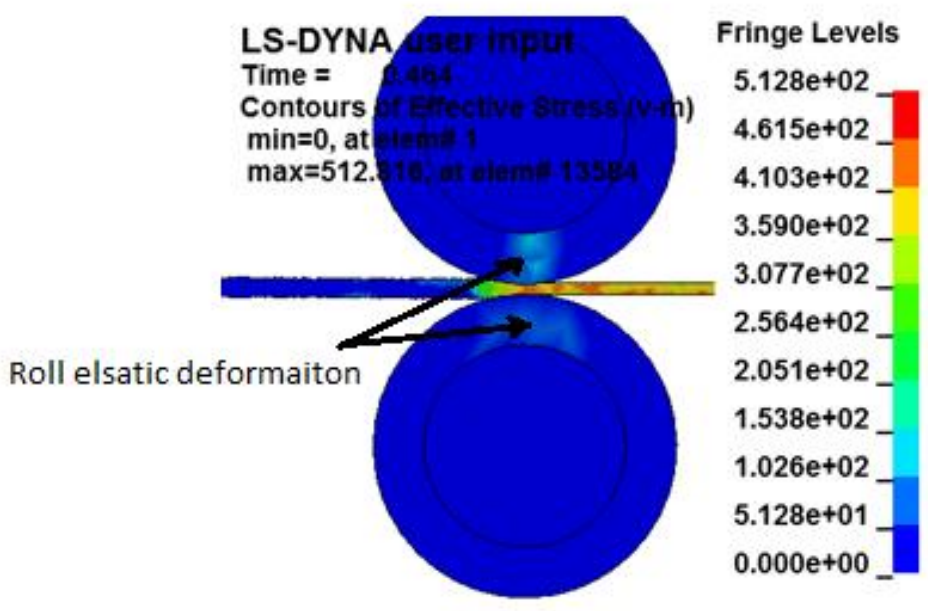

Fig. 13 Asymmetric rolling deformation.

Fig. 13 shows the rolling state in asymmetric deformation, the roller elastic deformation is shown in this figure. It can be seen that the maximum effective stress is around 512Mpa at time of $0.464 \mathrm{~s}$

\subsubsection{Rolling parameters effects}

The effective strain comparison between the elements in the central and the strip edge in Fig. 14. It can be seen that the effective strain is higher in the strip edge (1.5) than that in the central (1.0). This is because of the roller elastic deformation which results in an inhomogeneous rolling deformation across the strip width. More deformation exists in the strip edge which will be a potential factor for edge defect occurrence.

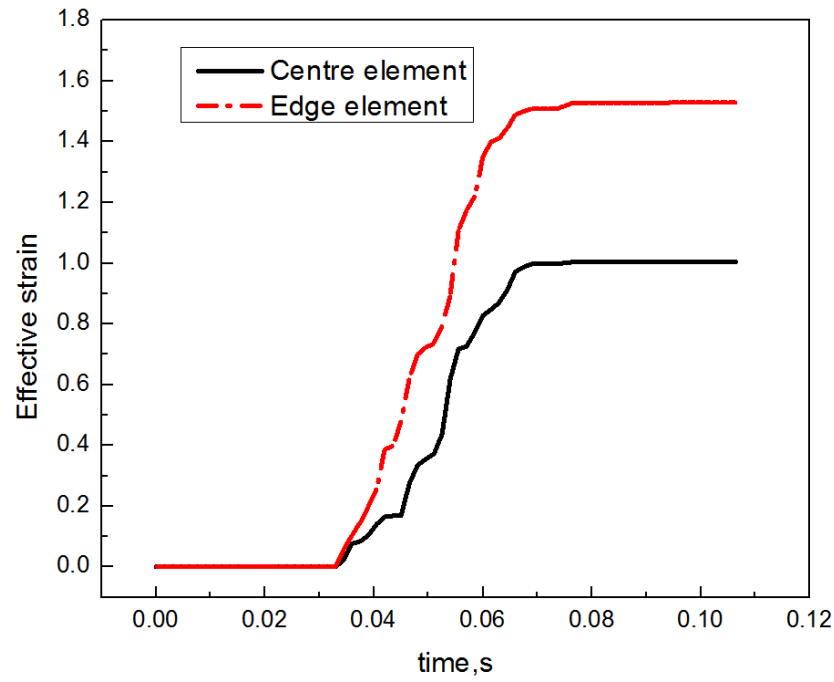

Fig. 14 Effective strain comparison between the element in the central and the strip edge.

Fig. 15 shows the rolling pressure under different roller speed asymmetric factor across the contact arc length. It can be seen that the maximum rolling pressure is in the position with contact length of $4 \mathrm{~mm}$. With an increasing of the speed asymmetric ratio, the maximum rolling pressure decreases. In addition, Fig. 15 reveals the cross shear zone length $m$ in contact arc length and the 
cross shear zone is longer in higher speed ratio than in low speed ratio. When the speed asymmetric ratio is 1 , the neutral points become a single one and the cross shear zone length $m$ is zero.

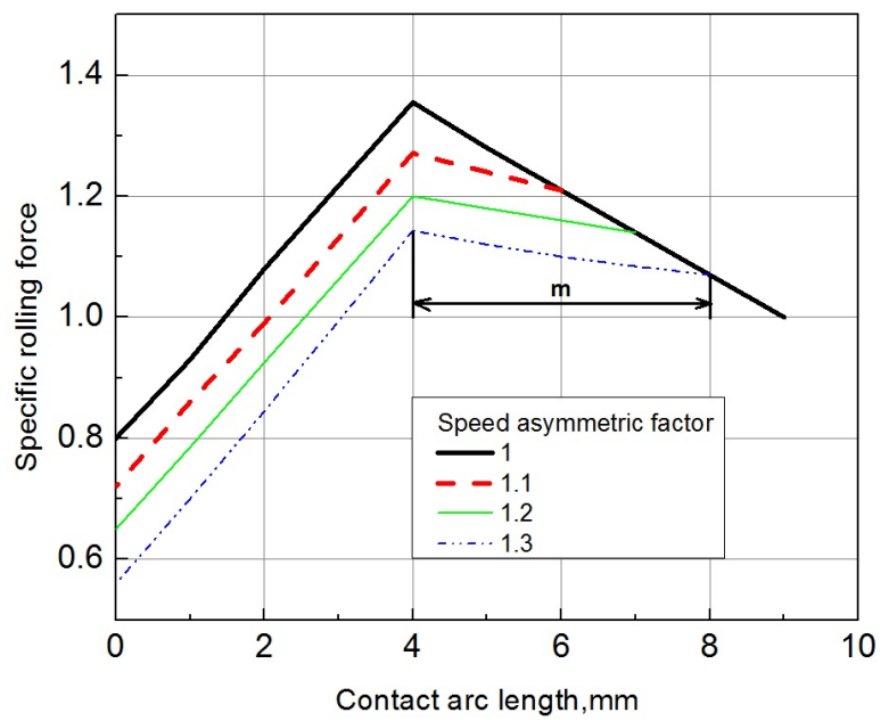

Fig. 15 Specific rolling pressure for various roller speed asymmetric factor.

( $m$ is cross shear zone length)

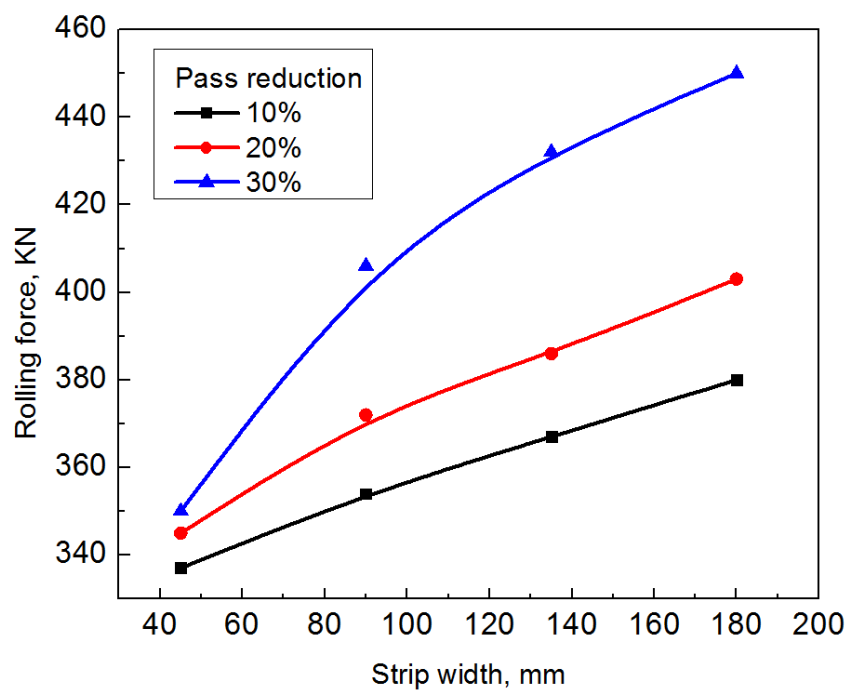

Fig. 16 Comparison of calculated rolling forces with strip width and reduction.

Comparison of the calculated rolling forces is shown in Fig. 16. Note that the calculated rolling force increases with the strip width and pass reduction. Fig. 17 shows the effect of speed asymmetric factor on specific rolling force across strip width. This verifies the feature of reducing the rolling force during asymmetric rolling. The specific rolling force along the strip width is nonuniform. For higher roll speed asymmetric factor, the specific rolling force at the edge is lower than that in lower speed asymmetric factor, which can reduce the contact between the roll and the rolled strip and reduce the work roll edge wear. 


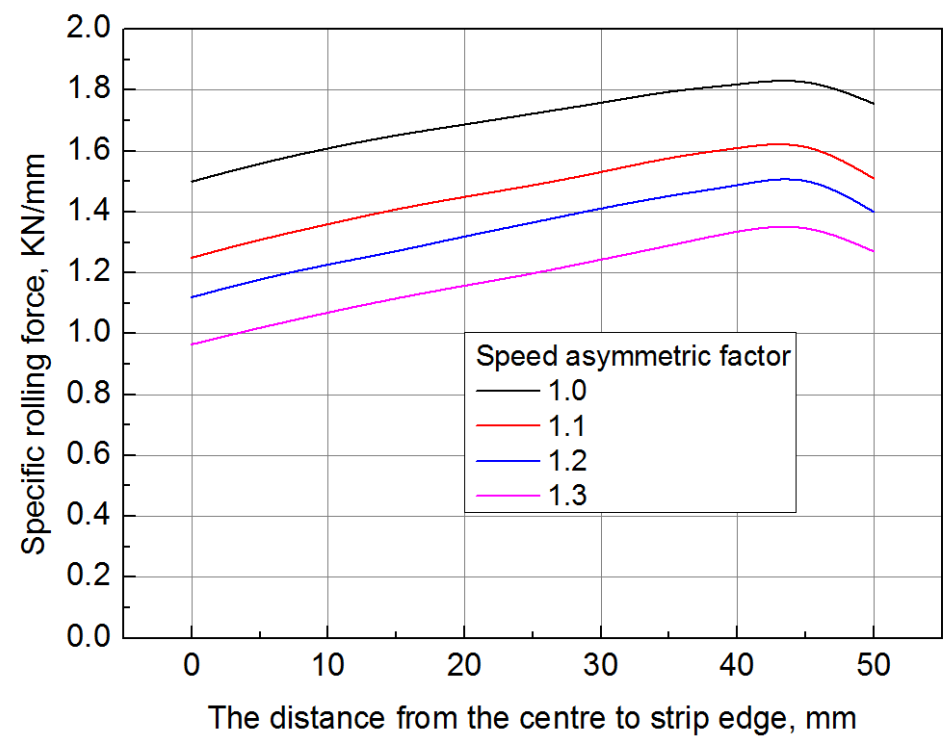

Fig. 17 Effect of speed asymmetric factor on specific rolling force across strip width.

\subsubsection{Strip curvature during asymmetric rolling}

Strip curvature in asymmetric rolling is an important evaluation item for strip shape quality. If the radius of outgoing curvature due to differences in the shear and axial strains are defined as $r_{1}$ and $r_{2}$, respectively, the resultant radius of curvature at exit is expresses as [30]

$$
\frac{1}{R_{e}}=\frac{1}{r_{1}}+\frac{1}{r_{2}}
$$

To distinguish between upward and downward curvature of the outgoing strip, the curvature index is defined as

$$
K=\frac{R_{e}-\frac{h_{0}}{2}}{R_{e}+\frac{h_{0}}{2}}
$$

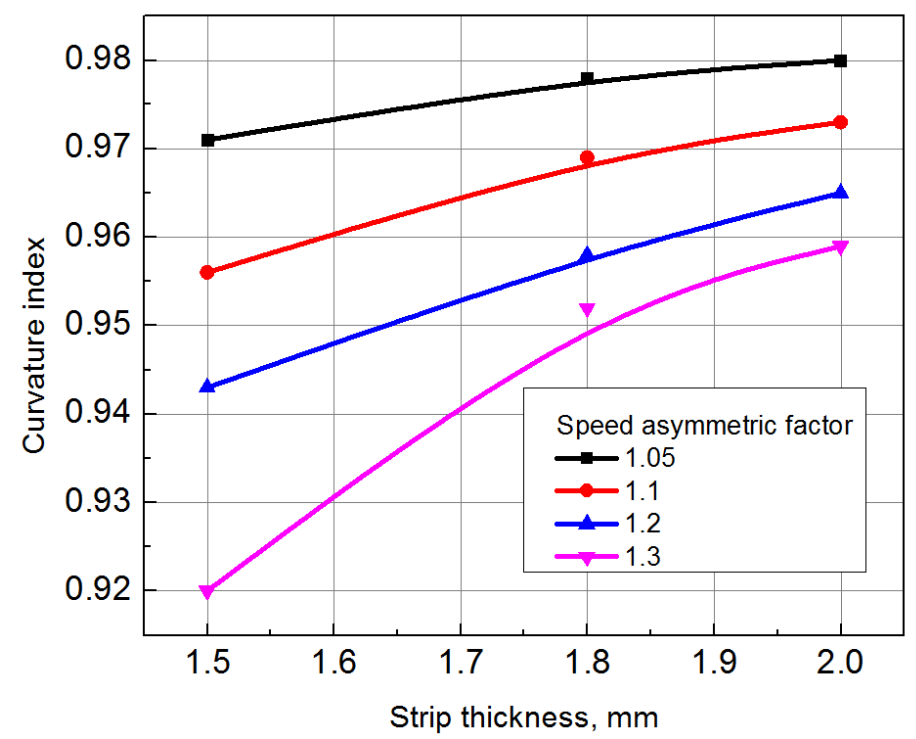

Fig. 18 Curvature index versus initial thickness for different speed asymmetric factor. 
In Fig. 18, variations of the curvature index for different input thicknesses at four specified speed asymmetric factors are depicted. As it is seen, in a small amount of speed mismatch, change in the initial thickness has no vigorous effect on the outgoing curvature. However, at high speed mismatch values, the curvature index effectively changes by increasing the initial thickness of the strip. The curvature index is always below to 1 and the simulation result shows that the strip bends always toward the lower-speed roll under this asymmetric rolling with equal diameter and different rotation velocity. This curvature affects the rolled strip shape profile and the applied forward tensile and backward tensile are benefit to reduce this curvature and maintain better strip shape profiles.

\section{Conclusions}

The FEM model has been proposed for the asymmetric rolling. The area of contact ratio increases as a function of specific rolling pressure. The surface roughness evolution has also been identified under various rolling conditions. The analysis shows that the strip bends toward the lower-speed roll under asymmetric rolling with equal diameter and different rotation velocity. Increasing the value of the speed asymmetry factor causes an increase in the strip curvature, while the force parameters decrease accordingly. The length of cross shear region in the roll bite increases and the neural points vary significantly with the speed asymmetric factor. The speed asymmetric factor also affects the specific rolling force across strip width significantly.

\section{Acknowledgements}

The first author is grateful to the Japan Society for Promotion of Science (JSPS), Japan for awarding of JSPS post-doctoral fellowship (FY 2012-2013) to carry out research in Japan and the support from Australian Academy of Science (AAS). This research was supported by the Grant-inAid for JSPS Fellows relating to JSPS Post-doctoral Fellowship for Foreign Researchers (Grant No. 24.02770) from JSPS.

\section{References}

[1] Yeong-Maw Hwang and Gow-Yi Tzou, Analytical and experimental study on asymmetrical sheet rolling, Int. J. Mech. Sci, 39 (1997) 289-303.

[2] M.P.F. Sutcliffe, Flattening of random rough surfaces in metalforming processes, J. Tribol. 121 (1999) 433-440.

[3] M.P.F. Sutcliffe, Surface asperity deformation in metal forming processes, Int. J. Mech. Sci., 30 (11) (1988) 847-868.

[4] H.R. Le and M.P.F. Sutcliffe, Analysis of surface roughness of cold-rolled aluminium foil, Wear 244 (2000) 71-78.

[5] Y. Kimura and T.H.C. Childs, Surface asperity deformation under bulk plastic straining conditions, Int. J. Mech. Sci., 41 (1999) 283-307.

[6] D.A. Korzekwa, P.R. Dawson and W.R.D. Wilson, Surface asperity deformation during sheet forming, Int. J. Mech. Sci., 34 (1992) 521- 539.

[7] S.W. Lo, T.C. Yang, Z.M. Shih and S.C. Lin, Effects of surface roughening on asperity flattening, Tribol. Lett., 35 (2009) 67-75.

[8] B. Bhushan, Contact mechanics of rough surfaces in tribology: multiple asperity contact, Tribol. Lett., 4 (1998) 1-35.

[9] B. Ma, A.K. Tieu, C. Lu and Z.Y. Jiang, Comparison of asperity flattening under different wavelength models for sheet metal forming, J. Mater. Process. Tech., 140 (2003) 635-640.

[10] P. Sadowski and S. Stupkiewicz, Combined effect of friction and macroscopic deformation, Tribol. Int., 43 (2010) 1735-1741.

[11] W.R.D. Wilson and S. Sheu, Real area of contact and boundary friction in metal forming, Int. J. Mech. Sci. 30 (1988) 475-489. 
[12] K. Manabe, T. Shimuzu, H. Koyama, M. Yang and K. Ito, Validation of FE simulation based on surface roughness model in micro-deep drawing, J. Mater. Process. Technol., 204 (2008) 89-93.

[13] L.F. Peng, X.M. Lai, H.J. Lee, J.H. Song and J. Ni, Friction behaviour modelling and analysis in micro/meso scale metal forming process, Mater. Design, 31 (2010) 1953-1961.

[14] Handbook of Micro/Nanotribology, Edited by Bharat Bhushan and Boca Roton: CRC Press LLC, 1999.

[15] T.S. Yang and S.W. Lo, Contact simulation for predicting surface topography in metal forming, Tribol. Lett., 23 (2006) 121-129.

[16] G.Y. Kim, M. Koc and J. Ni, Experimental and numerical investigations on microcoining of stainless steel 304, J. Manuf. Sci. Eng., ASME, 130 (2008) 041017-1/6.

[17] S.C. Pan, M.N. Huang, G.Y. Tzou and S.W. Syu, Analysis of asymmetrical cold and hot bond rolling of unvounded clad sheet under constant shear friction, J. Mater. Process. Technol., 177 (2006) 114120.

[18] V. A. Nikolaev and A. A. Vasilyev, Analysis of Strip Asymmetrical Cold Rolling Parameters, Metall. Min. Ind., 2(2010) 405-412.

[19] F. Farhat-Nia, M. Salimi and M.R. Movahhedy, Elasto-plastic finite element simulation of asymmetrical plane rolling using an ALE approach, J. Mater. Process. Technol., 177 (2006) 525-529.

[20] M. Qwamizadeh, M. Kadkhodaei and M. Salimi, Asymmetrical sheet rolling analysis and evaluation of developed curvature, Int. J. Adv. Manuf. Technol. 61 (2012) 227-235.

[21] H.B. Xie, Z.Y. Jiang and W.Y.D. Yuen, Analysis of friction and surface roughness effects on edge crack evolution of thin strip during cold rolling, Tribol. Int., 44 (2011) 971-979.

[22] R.M. Patrikar, Modeling and simulation of surface roughness, App. Surf. Sci., 228 (2004) 213-220.

[23] Methods for generating rough surfaces in ANSYS, M. Kathryn Thompson, Mechanical Engineering Department, MIT.

[24] J. A. Greenwood and G.W. Rowe, Deformation of surface asperities during bulk plastic flow, J. Appl. Phys. 36 (1965) 667-668.

[25] H. Keife and C. Sjogren, A friction model applied in the cold rolling of aluminium strips, wear 179 (1994) 137-142. 